\title{
DARRIEUS TYPE WIND TURBINE WITH CONTROLLED BLADES
}

\author{
Kayan V., Lebid O. \\ Institute of Telecommunications and Global Information Space of the \\ National Academy of Sciences of Ukraine, Kyiv, Ukraine, kayan@ua.fm
}

\begin{abstract}
The results of studies on possibility of increasing efficiency in the use of wind energy and improving the dynamic characteristics of Darrieus wind turbine with straight blades are described. It is shown how the values of torque on the rotor shaft may be optimized by controlling the orientation of the turbine blades relative to the oncoming flow. Control of blades was provided with a cylindrical track of the special form in plan. The track form allowed to establish optimum angle of attack on each site of blade circular trajectory. It allowed to increase power coefficient Cp by 1.5 times and to reduce wind speed at which there is self-start of the wind turbine.
\end{abstract}

Keywords: Darrieus type wind turbine, controlled blades, torque, power coefficient, tip speed ratio

\section{Introduction}

As it is known, all over the world, the technologies have been intensively developed using renewable energy sources, including kinetic energy of wind and water flows [1]. The main types of the wind power units are the wind generators with a horizontal or vertical axis of rotation, the electric generator for converting mechanical energy on the turbine shaft into electric and the tower for the placement of these generators. At present, for utilization of wind energy the horizontal axis wind turbines (HAWT) with capacity from hundreds watts to several megawatts are widely used. The essential components of such wind turbine construction is the vertical tower with height from 10 to 150 meters, the mechanisms for orientation the area of wind turbine rotation perpendicular to the direction of wind flow.

Vertical axis wind turbines (VAWT) do not need special arrangements of orientation relative to the direction of the wind and allow two-bearing turbine mounting system. The electric generator may be placed in the base of the unit, which simplifies its construction. The important advantage of VAWT is the relative simplicity of blades construction and the relatively small area required for the placement of wind turbines [2]. One of the main disadvantage of Darrieus type VAWT with rigidly fixed blades relative to the horizontal crosspieces is a high-speed wind flow, at which there is the self-starting of VAWT to rotate, and large values of the variable mechanical load on the shaft. As it turned out both of these disadvantage can be eliminated by using special methods of control the position of the blades on its circular trajectory.

\section{Darrieus type VAWT design with straight controlled blades}

When moving in a circular path the blade of VAWT operates in a periodically changing unsteady flow. As the typical wing profiles are used in the construction of blades, the main parameter determining the value and direction of the forces acting on the profile is the angle of attack. The nature of blades movement in the turbine with fixed position of blades is such that the angle of attack becomes supercritical at a very large part of its trajectory [3]. This leads to flow stall and big decrease of the value of the useful component of the aerodynamic forces, so that the blade even brakes wind turbine in some parts of the trajectory.

However, if it is possible to turn the blade in such a manner that the flows are at the optimal angle of attack around of the blade profile, the produced value of torque on the VAWT shaft can be significantly increased. The test of turbine models in water [3-4] and the full-scale prototypes of VAWTs in the air [4-5] have shown considerable increase the power coefficient

$$
C_{p}=2 P / \rho V^{3} S,
$$

where $P$ - is capacity of wind turbine, $\rho$ - density of air or water, $V$ - velocity of air or water flow, $S$ - swept area of wind turbine) as well as a significant decrease in the average load on the turbine shaft and its amplitude pulsations [5-6]. 
The last prototype of the series of the wind turbines was created and tested in 2013-2018 and showed a stable and reliable performance during the tests in the wind tunnel, and the value of coefficient $C_{p}$ was received equal 0.45. Darrieus type VAWT with straight controlled blades (Fig. 1) had the following parameters: blade length $l_{\text {blade }}=1.6 \mathrm{~m}$, the chord length of the blade $b=0.25 \mathrm{~m}$, the profile of the blade is symmetrical NACA 0015, the blade aspect ratio $\mathrm{AR}=l_{\text {blade }} / b=6.4$, radius of blade rotation $R=0.7 \mathrm{~m}$, the average diameter of the control track $D=0.4 \mathrm{~m}$, the VAWT swept area $S=2 R l_{\text {blade }}=2.24 \mathrm{~m}^{2}$, the solidity $\sigma=$ $3 b / 2 R=0.54$. The blades were made of carbon plastic and one blade weighed $2.7 \mathrm{~kg}$.

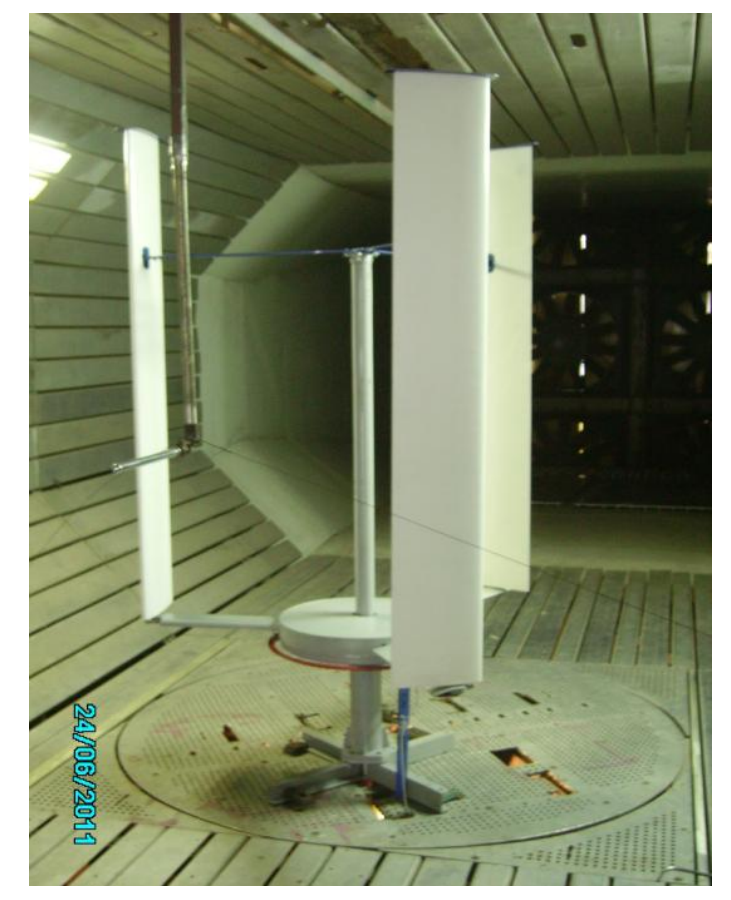

Fig.1. The wind turbine with controlled blades in the wind tunnel

Mechanism for blades control have cylindrical track of the special form in plan and $8 \mathrm{~mm}$ thick, placed below the lower crosspieces of the VAWT, on both sides of which three pairs of rollers moved. The pair of rollers connected by special carriages, which are in its turn were pivotally connected to the rods disposed within the lower horizontal crosspieces. Another ends of the rods were pivotally connected to the control axes at the lower end of the blades. The control track was attached to the bottom support rigidly. To determine the shape of the cylindrical track was used computer modeling of the flow around the VAWT. A modified method of discrete vortices was used for modeling. This method based on the model of inviscid incompressible fluid [7]. A variable nature of the flow around blades is a feature of this modification: a flow around blade is unseparated at subcritical angles of attack of blade, at supercritical angles of attack a flow separate on leading edge of blade, as shown in [8].

\section{Results and discussions}

The used method of computer modeling allows to view the time evolution of the vortex trail behind VAWT and to get instantaneous and integral dynamic characteristics of VAWT. The presence of numerical flow visualization helps to better understand the physics of the processes and identify patterns that cannot be identified solely by instrumental measurements.

The results of numerical visualization of a vortex flow behind VAWT with fixed $(a)$ and controlled $(b)$ blades at tip speed ratio $\lambda \mathrm{p}=2 \pi \mathrm{n} R / V=1.2$ are shows in Fig.2 (the clockwise-rotating vortices are blue, counterclockwise rotating vortices are red, more intensive vortices are brighter). It can be seen that for VAWT with fixed blades the trail has a pronounced turbulent character that adversely affects the dynamic characteristics of the VAWT. For the VAWT with controlled blades highly visible vortex trail is formed and this trail leads to the improvement of dynamic characteristics of the VAWT. Note that such a marked difference vortex trail occurs precisely at tip speed ratio $\lambda \mathrm{p}=1.2$, which was selected for controlling track 
optimization. By change $\lambda \mathrm{p}$ in upward or downward from this value the vortex trail behind the VAWT quickly loses its orderly appearance and the positive effect of the control blades loses also.

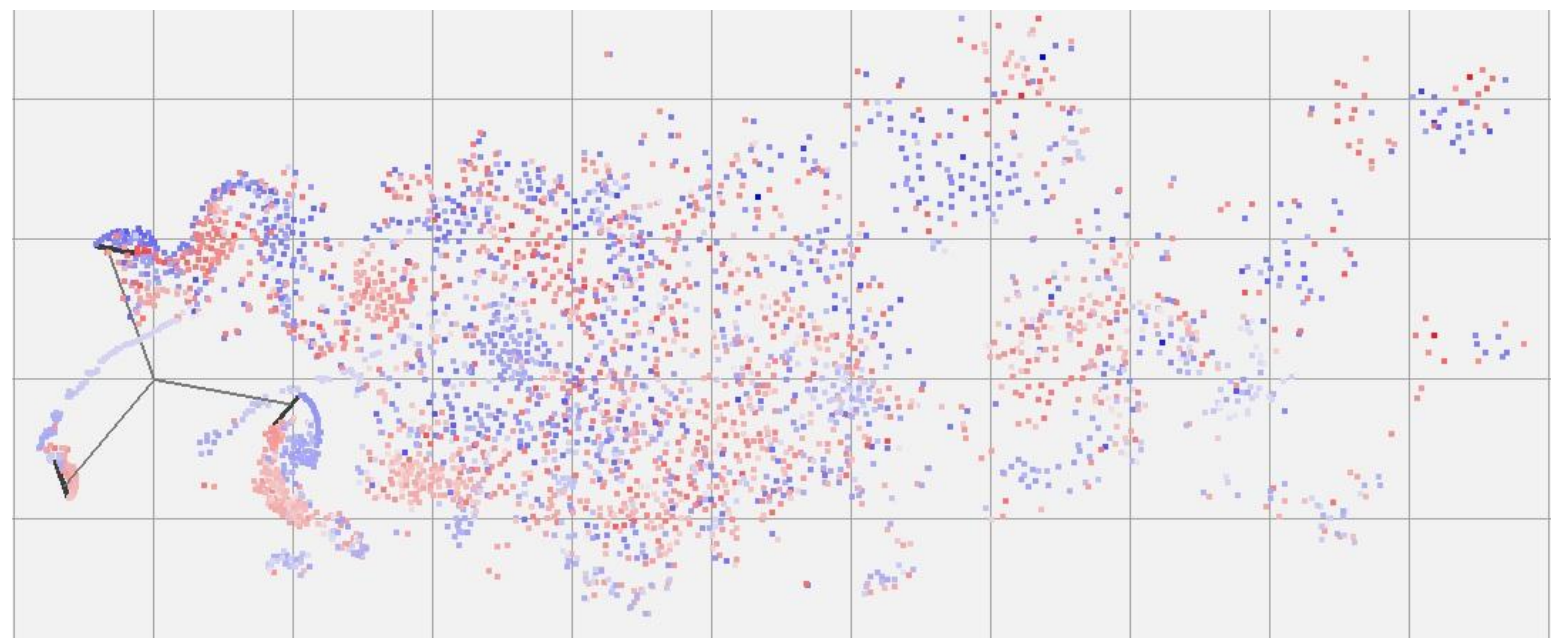

$a$

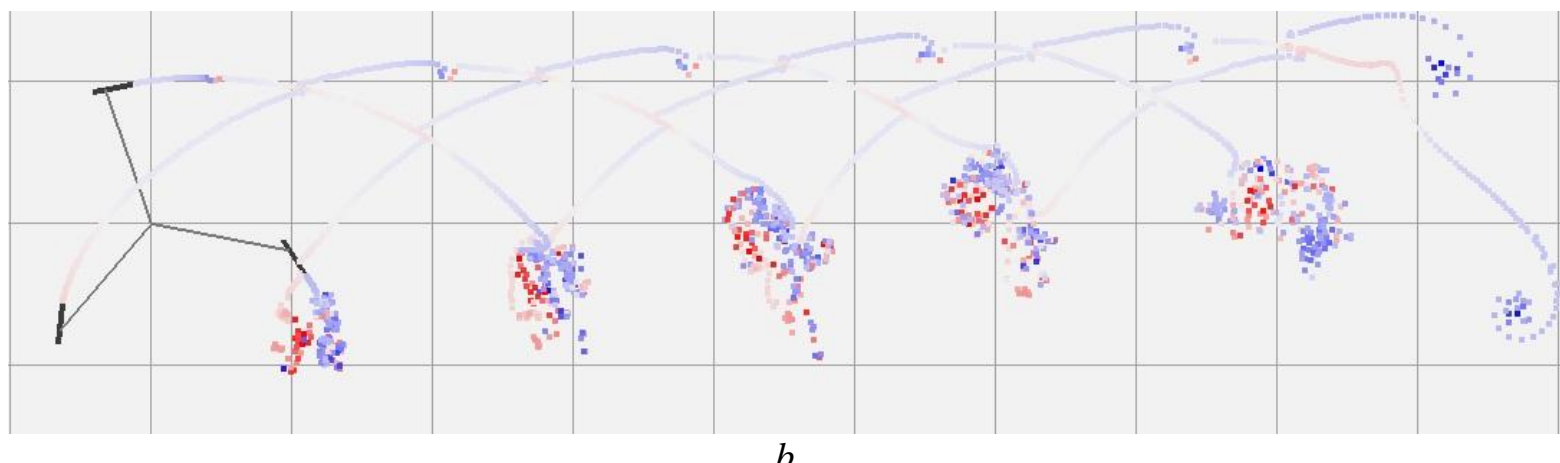

Fig. 2. Numerical visualization of a vortex flow behind VAWT with fixed $(a)$ and controled $(b)$ blades at tip speed ratio $\lambda \mathrm{p}=1.2$

The lower end of the turbine shaft went under the floor of the wind tunnel, where on the shaft the disc with the 60-hole for measuring the speed of rotation of VAWT was placed on the shaft, and the shaft through gear box with conic gears connected to the electromagnetic powder brake coupling. The range of brake torque of coupling is $3 \div 80 \mathrm{Nm}$ in increments of $2 \mathrm{Nm}$. Under the initial load on the shaft of $3 \mathrm{Nm}$ (resistance coupling with the power supply disconnected), VAWT self-started when the wind flow velocity in wind tunnel was $V=2.5 \div 2.8 \mathrm{~m} / \mathrm{sec}$, without load (i.e. the coupling is removed) the VAWT self-started when the wind flow velocity was $V=1.8 \div 2 \mathrm{~m} / \mathrm{sec}$. Wind flow velocity $V$ was defined by the stationary of wind tunnel equipment with an accuracy of $0.05 \mathrm{~m} / \mathrm{sec}$. The same VAWT without control mechanism (i.e. the blades are rigidly fixed to the crosspieces) and without load on the shaft self-started when the wind flow velocity $V=3.5 \div 3.8 \mathrm{~m} / \mathrm{sec}$. Dependence of the rotation speed of the VAWT $\boldsymbol{n}$ from the useful torque $M_{n e t}$ on the shaft at various velocity of wind flow $V$ is shown in Fig. 3, $a$. The useful torque $M_{n e t}$ was set by change of current in electromagnetic brake coupling. The full torque $M_{\text {full }}$ created by the wind turbine at rotation in a wind stream was defined as the sum of useful torque $M_{n e t}+$ drag moments of structural elements of the wind turbine and the braking moment during the control mechanism operation, which defined experimentally at various speeds of turbine rotation without blades when $V=0$.

The maximum power on shaft was obtained at the lowest rotation speeds of the VAWT. The control of blades allows to get a torque on the shaft almost three times superior than the VAWT with blades rigidly fixed, thus almost twice the speed of rotation decreases (Fig. 3, $a$ ). This is clearly seen in Fig. 3, $b$, where the maximum of power coefficient $C_{p}$ of the VAWT with controlled blades obtained by tip speed ratio $\lambda p=1.1$, and the maximum of power coefficient $C_{p}$ of the VAWT with blades which are rigidly fixed obtained by $\lambda p=$ 
2.1. At the same time the power coefficient $C p_{n e t}$ of the VAWT with controlled blades almost 1.5 times higher than the same with rigidly fixed blades.

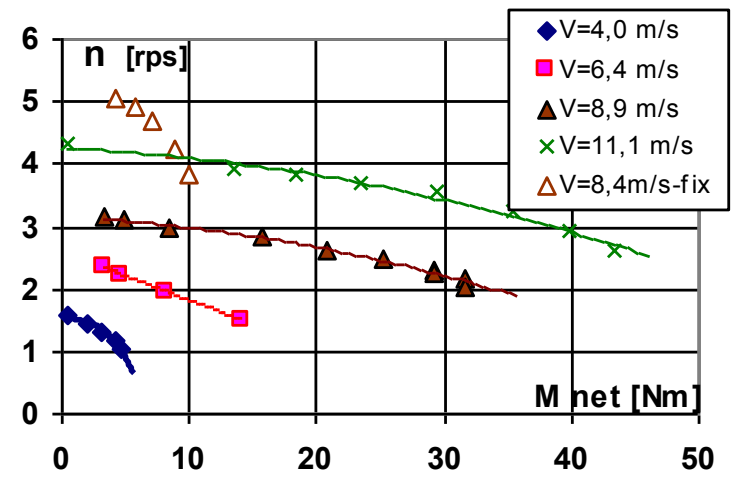

$a$

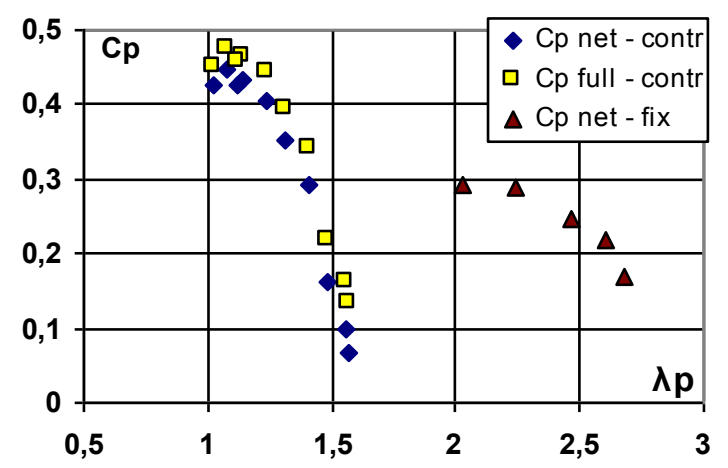

$b$

Fig. 3. The dependences of the VAWT rotation speed $\boldsymbol{n}$ from the torque $M_{\text {net }}$ on the shaft at various speeds of wind flow $V(a)$ and the dependence of the energy coefficient $C_{p}$ from the value of tip speed ratio $\lambda p(b)$ at wind flow $V=9 \mathrm{~m} / \sec \left(C_{p-\text { contr }}-\right.$ controlled blades, $C_{\mathrm{p}-\text { fix }}-$ fixed blades $)$.

At rigid attachment of blades the angle of the blades placement on the crosspiece was $8^{\circ}$, which agrees with the data obtained at the Institute of McMaster in Canada [9] while purging of the VAWT with sizes $l_{\text {blade }}$ x $D=3 \times 2.5 \mathrm{~m}\left(C p_{f u l l}\right.$ was received experimentally and was not more than 0.33$)$.

Thus, the use of simple constructive and technological control blades mechanism of the wind turbine with vertical axis allows using it effectively in small $(6-8 \mathrm{~m} / \mathrm{sec})$ wind speeds, reducing the value of wind load on the wind turbine shaft, and significantly improving the performance characteristics.

\section{Conclusions}

The Darrieus type wind turbine with straight controlled blades was designed and was built. The results of turbine tests in wind tunnel approved the efficiency of this turbine as the wind or water energy converter is 1.5 times higher than the best world samples. The electric generator model was composed for wind power unit based on VAWT with controlled blades. The calculated results were compared to experimental results. The mean difference between these results is not higher than $7 \%$.

\section{REFERENCES}

1 Sakipova S., Jakovics A. Sail-type wind turbine for autonomous power supply: Possible use in Latvia. Latvian Journal of Physics and Technical Sciences No 51(6), 2014, pp. 13-25.

2 Avallone E. A., Baumeister T., Sadegh A. M., Marks Standard Handbook for Mechanical Engineers. 11-th Ed., McGraw Hill. New York, 2006, 685p.

3 Kayan V., Dovgy S., Lebid O. Darrieus Water Turbine with Active Control of Blades Prospective Renewable Power Generation Device for Slow Moving Water. Book of Abstracts of the 6th Conference on Sustainable Development of Energy, Water and Environment Systems, Dubrovnik, Croatia, 2011, A-217, pp. 376-377.

4 Kayan V., Darrieus Turbine with Controlled Blades: The Perspective Converter of Hydrokinetic Energy. Open Journal of Renewable Energy and Sustainable Development, CA, USA, 2014, Vol.1, No.2, pp. 9 - 23.

5 Kayan V., Kochin V., Lebid O., Studying the Performance of Vertical Axis Wind Turbine Models with Control Mechanism of Blades. Intern. Journal of Fluid Mechanics Research, USA, 2009, Vol. 36, No. 2, pp. 154 - 165.

6 Kayan V. P., Lebid O. G., Performance optimization of full-scale Darrieus type wind turbine with straight controlled blades. Applied hydromechanics (Ukraine), 2010, No. 4, pp. 26 - 35.

7 Cottet G.-H., Koumoutsakos P.D. Vortex Methods: Theory and Practice. Cambridge U. Press, 2000, 314p.

8 Gorban I.M., Lebid O.G. Numerical Modeling of the Wing Aerodynamics at Angle-of-Attack at Low Reynolds Numbers. In: Sadovnichiy V. Zgurovsky M. (eds) Modern Mathematics and Mechanics. Understanding Complex Systems. Springer, Cham., 2019, pp. $159-179$.

9 Fiedler A. J., Tullis S., Blade Offset and Pitch Effects on a High Solidity Vertical Axis Wind Turbine. Wind Engineering, 2009, Vol. 33, No 3, pp. $237-246$. 